Classification

Physics Abstracts

$68.10-47.25 \mathrm{Q}-44.25-91.35$

\title{
What is responsible for thermal coupling in layered convection?
}

\author{
H. C. Nataf, S. Moreno and Ph. Cardin \\ Laboratoire de Géophysique, Bât. 510, Université Paris-Sud, 91405 Orsay Cedex, France
}

(Reçu le 18 février 1988, révisé le 14 mai 1988, accepté le 13 juin 1988)

\begin{abstract}
Résumé. - Nous avons mené des expériences de laboratoire sur la convection dans un système bicouche. Le système est formé de deux couches liquides superposées, de même épaisseur. Les liquides sont immiscibles : il s'agit d'huile au silicone en haut, et de glycérol en bas. Nous avons analysé la structure convective et obtenu des données pour le champ de température et celui de vitesse. Nous trouvons que le couplage entre les deux systèmes convectifs est "thermique ", c'est-à-dire que les cellules de convection se correspondent, et que les courants montants dans la couche du haut sont au-dessus des courants montants de la couche du bas. Ce résultat est surprenant car il est en contradiction avec les résultats d'expériences numériques récemment obtenus sur la convection bicouche. Les expériences numériques montrent que le couplage « mécanique " (les cellules se correspondent mais tournent en sens opposé) est le mode stable dans les conditions que nous avons essayé de reproduire en laboratoire. Nous avons mené une série de tests pour essayer d'isoler le phénomène qui est responsable du désaccord entre les deux types d'analyse. Nous proposons un éventuel mécanisme qui fait appel à une viscosité interfaciale longitudinale, dont l'origine n'est pas encore élucidée.
\end{abstract}

\begin{abstract}
Laboratory experiments have been conducted on convection in a layered system. The system consists in two liquid layers of equal thickness. The liquids are immiscible : the upper one is silicon oil, and the lower one is glycerol. The structure of convection has been analysed, and data obtained both on the temperature field and the velocity field. It is shown that the coupling between the two convecting systems in «thermal », i.e. convection cells are superposed with uprising currents above uprisings. This result is surprising because it contradicts numerical experiments recently obtained for layered convection. These find « mechanical » coupling (cells are superposed but turn in opposite senses) to be the stable mode for the conditions we tried to reproduce in the laboratory. Several tests have been conducted in order to isolate the phenomenon which is responsible for the discrepancy between the two types of analyses. A tentative mechanism is proposed: it involves an equivalent interfacial longitudinal viscosity, whose origin is not yet clearly understood.
\end{abstract}

\section{Introduction.}

Whether convection in the Earth's mantle is « whole mantle » or «layered», with the upper mantle convecting separately on top of the lower mantle, is still a debated issue $[1,2]$. Nevertheless, the fact that the question arises has prompted geophysicists to study 2-layer convection is some detail [2-4]. Physicists have recently become interested in this problem as well $[5,6]$.

In this paper, we will concentrate on the type of coupling that exists between the two convecting layers. Two end-member types of coupling have been recognized : «mechanical coupling " and « thermal coupling ", which are schematically shown in figure 1. Most geophysical studies ignore inertial effects and surface tension, since both are negligible in the Earth's mantle. Within this frame, marginal stability has been discussed by Richter and Johnson [3], and Honda [7]. Both find that «mechanical coupling " is preferred once the density jump at the interface is large enough. Richter and McKenzie [2] present numerical experiments that agree with the previous results. Cserepes and Rabinowicz [4] have conducted the most thorough numerical analysis. They also find "mechanical coupling " to be the preferred mode when the viscosities of the two fluids are of the same order. When the viscosities are very different the viscous coupling at the interface is reduced, and «thermal coupling» takes over. Cserepes and Rabinowicz find that a viscosity ratio of 30 is needed for this to occur. Cserepes et al. [8] have recently continued their analysis by performing three-dimensional numerical experiments. These ex- 
(a)

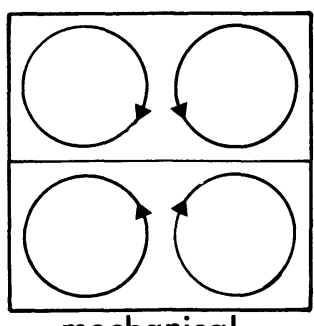

(b)

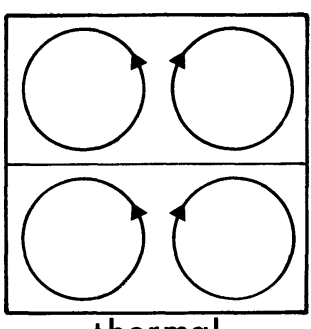

thermal

Fig. 1 - Sketch of two possible types of coupling in a twolayer convecting system : (a) mechanical coupling : rolls turn in the opposite sense, so as to minimize the shear at the interface ; $(b)$ thermal coupling : hot uprisings in the top layer correspond to hot uprisings in the bottom layer.

periments nicely confirm their previous findings. The wealth of the available numerical results made it tempting to compare them with precise laboratory experiments. Previous laboratory experiments $[2,9]$ were done using miscible fluids. In that case, a diffusion layer builds up at the interface and can be entrained into the fluids. The entrained «filaments " seem to play an important dynamical role, which is difficult to control in the lab. These studies did not report on the prevailing type of coupling. In the present experiments, we used immiscible fluids in order to eliminate the entrainment of filaments. More specifically, we concentrated on experiments that were as close as possible to cases already treated numerically by Cserepes and Rabinowicz [4]. It quickly appeared that the type of coupling we obtain in the lab is «thermal coupling », in marked disagreement with the numerical results. The next section gives a brief description of the experimental set-up. The evidence for "thermal coupling " is exposed next. Finally, we examine what could be the reason for the discrepancy between the numerical and the laboratory experiments. A tentative mechanism is proposed, but the question remains largely open.

\section{Experimental set-up.}

The layers are two immiscible fluids, silicon oil above glycerol, whose properties are listed in table I. They are enclosed in a frame made of $30 \mathrm{~mm}$-thick lucite walls sandwiched between two horizontal copper plates. The inner dimensions of the frame are $50 \mathrm{~mm}$ (height) $\times 250 \times 125 \mathrm{~mm}$. Each of the copper plates is maintained at a given temperature, which is monitored using thermocouples. Horizontal variations are less than $0.15^{\circ} \mathrm{C}$, and time fluctuations less than $0.1{ }^{\circ} \mathrm{C}$. The tank is mounted on a moving base that allows translation in the 3 directions of space $(X, Y, Z)$. The displacements are produced by computer-controlled stepping motors, with a precision of $0.1 \mathrm{~mm}$. Figure 2 schematically shows the different methods we use to observe the temperature and velocity fields. Differential interferometry gives an instantaneous image of the horizontal or vertical gradients of temperature in part of the $X Z$ -

Table I. - Fluid properties.

Units

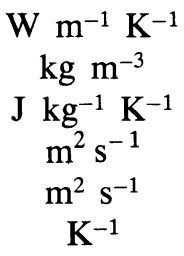

\footnotetext{
$\alpha$ coefft of thermal expansion

index of refraction

$S$ interfacial tension $\left(25^{\circ} \mathrm{C}\right)$

$\sigma$-temperature derivative of $\mathrm{S}$

Pr Prandtl number $=\nu / \kappa\left(25^{\circ} \mathrm{C}\right)$

$\rho$ density $\left(25^{\circ} \mathrm{C}\right)$

$C_{\mathrm{P}}$ specific heat

$\kappa$ thermal diffusivity

$\nu$ kinematic viscosity $\left(25^{\circ} \mathrm{C}\right)$
}

$\mathrm{N} \mathrm{m}^{-1}$

$\mathrm{N} \mathrm{m}^{-1} \mathrm{~K}^{-1}$
Glycerol $98 \%$

( $\left.{ }^{1}\right)$
Silicon oil $\left({ }^{2}\right)$

Rhodorsil 47V500
0.294

0.16

$1.26 \times 10^{3}$

$2.62 \times 10^{3}\left({ }^{3}\right)$

$0.89 \times 10^{-7}$

$0.97 \times 10^{3}$

$1.46 \times 10^{3}$

$1.13 \times 10^{-7}$

$4.99 \times 10^{-4}\left({ }^{4}\right)$

$9.45 \times 10^{-4}$

$4.9 \times 10^{-4}$

1.403

1.475

$25 \times 10^{-3}\left({ }^{5}\right)$

$-1.3 \times 10^{-4}(5)$

8400

4400

(1) From « Handbook of Physics \& Chemistry », 1967, unless otherwise specified.

( ${ }^{2}$ ) From Rhône Poulenc technical release, unless otherwise specified.

( ${ }^{3}$ ) From « Handbook of Tables for Applied Engineering Science », 1970.

${ }^{(4)}$ Measured, using a Haake falling ball viscometer.

(5) Measured by arrachment, using a Prolabo/Schlumberger tensiometer. 


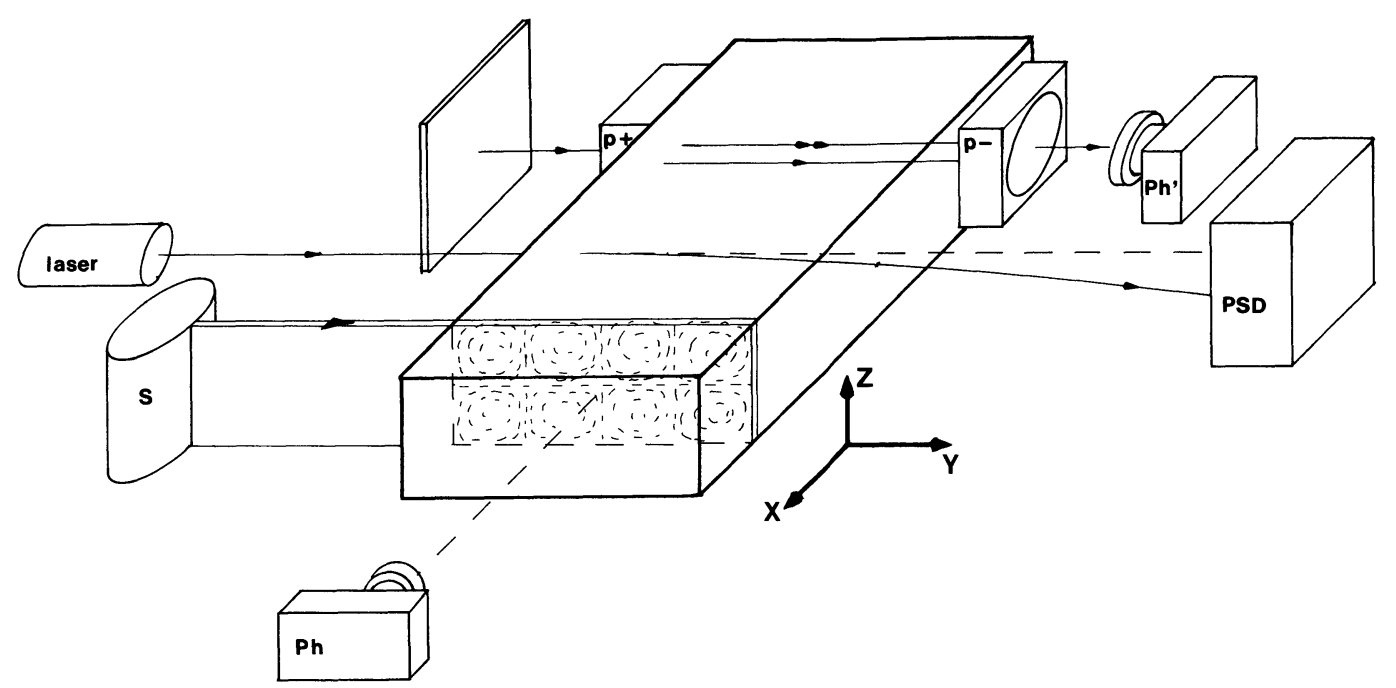

Fig. 2 - Schematic perspective view of the different experimental methods. Differential interferometry: fringes of interference are observed in camera Ph'. Every incident ray is split into two parallel rays by the Savart-Françon polariscope $\mathrm{p}+$ and recombined in $\mathrm{p}-$. Strioscopy : the deflection of the laser beam after it has crossed the tank is monitored on the position sensitive photodetector PSD. Velocity mapping : a vertical slice of fluid is illuminated by the slot $\mathrm{S}$; streak photographs are taken in $\mathrm{Ph}$.

plane [10]. Strioscopy is based upon the deflection of a laser beam by the temperature gradients it encounters when crossing the tank. It is a local, yet $Y$ integrated, measurement. The deflection is measured on a $10 \times 10 \mathrm{~mm}$ position-sensitive photodetector, with a precision of about $0.1 \mathrm{~mm}$. Since the tank can move in all directions, $X$ or $Z$ profiles are easily obtained. The two methods just described are insensitive to variations in the $Y$-direction. In order to observe the structure of convection parallel to the long direction of the tank, we use aluminum flakes as velocity tracers. A vertical $Y Z$ slice of fluid is illuminated, and the camera stands on the side. The color of the light source is changed according to some known time-scenario, so that both the amplitude and the sense of the velocities can be reliably obtained from the streak photographs. Finally, the deformation of the interface is measured by monitoring the position of a laser spot that is reflected off the interface, for various $Y$ positions.

\section{Evidence for thermal coupling.}

Most experiments described here are performed with two layers of equal thickness. The Rayleigh numbers for each layer range from 5000 to 25000 . With these conditions, numerical experiments find that only «mechanical coupling » is possible $[4,7$, 8], even when the top and bottom boundaries are rigid as in the laboratory [11]. However, we systematically observe "thermal coupling " in the lab.

An example is given in figure 3 . The Rayleigh numbers $\left({ }^{1}\right)$ and other useful parameters for this experiment, numbered $\mathrm{N} 3$, are given in table II.
Two rolls are visible in each layer within the view field of the differential interferometry image. A cold downwelling current is visible in the upper layer in the center of the picture. It stands above a cold downwelling current of the lower layer. The coupling is therefore clearly «thermal».

Using strioscopy, we can check that this situation prevails everywhere in the tank. Figure 4 presents $X$-profiles of the horizontal gradient of temperature taken at mid-depth of each layer for experiment N5. Each zero-crossing marks an uprising or a downwelling current. Rolls are clearly superposed and « in phase », indicating «thermal coupling » again. The shape and amplitude of the temperature gradient profiles indicates that convection is slightly more vigorous in the upper layer, as expected from the Rayleigh numbers evaluated in table II.

TRANSVERSE ROLLS. - The two results presented so far ignore the structure in the $Y$-direction. We found however that, although the Rayleigh numbers are fairly small, the convection pattern is usually bimodal. For the lowest Rayleigh numbers there is only one set of rolls but they tend to form with their axis parallel to the long side of the box. It is therefore important to observe the structure in the

( $\left.{ }^{1}\right)$ In order to facilitate the comparison with usual Rayleigh-Bénard convection, we define Rayleigh numbers for each individual layer. These are not really external parameters, since the temperature at the interface is not known a priori. The interface temperature used in table II is simply the temperature there would be in the conductive state. Other non-dimensional groups can be calculated using material properties in table I. 

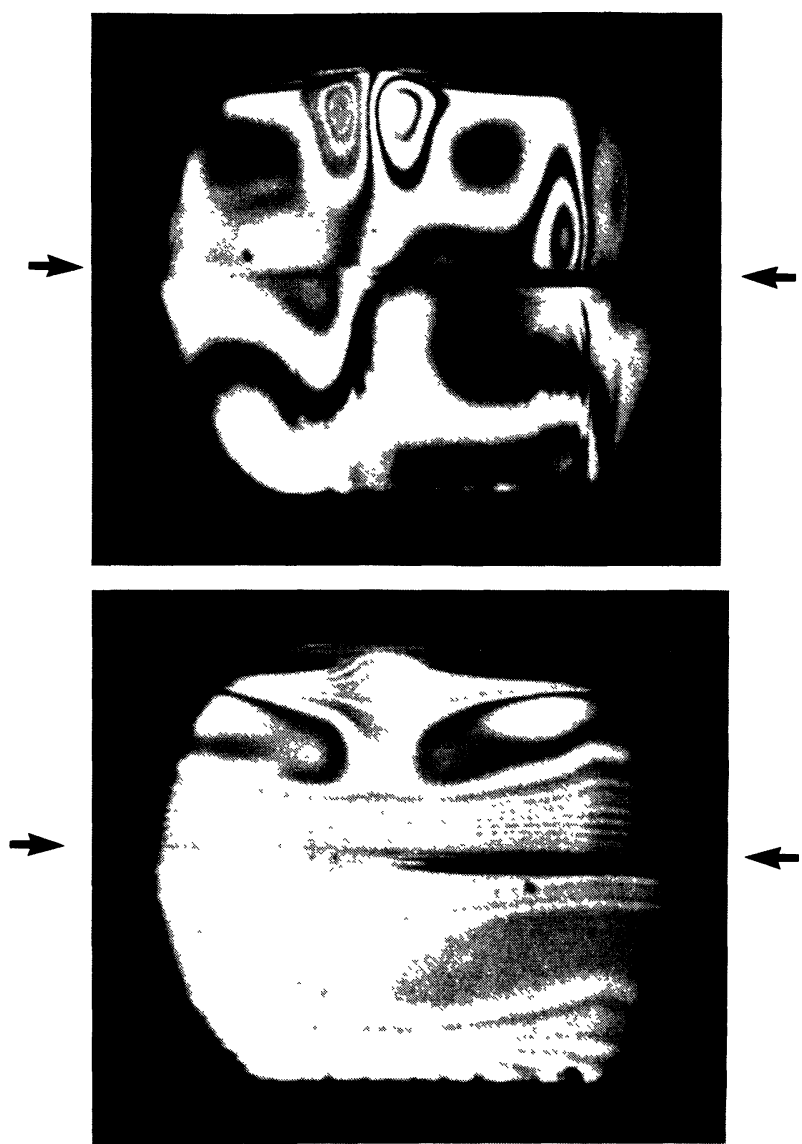

Fig. 3 - Pictures of the horizontal (top) and vertical (bottom) gradient of temperature for experiment N3. The arrows indicate the position of the interface. The two « eyes " in the top of the upper layer indicate a downwelling current [10]. A less distinct but similar pattern in the lower layer reveals that there is a downwelling in this position in that layer as well.
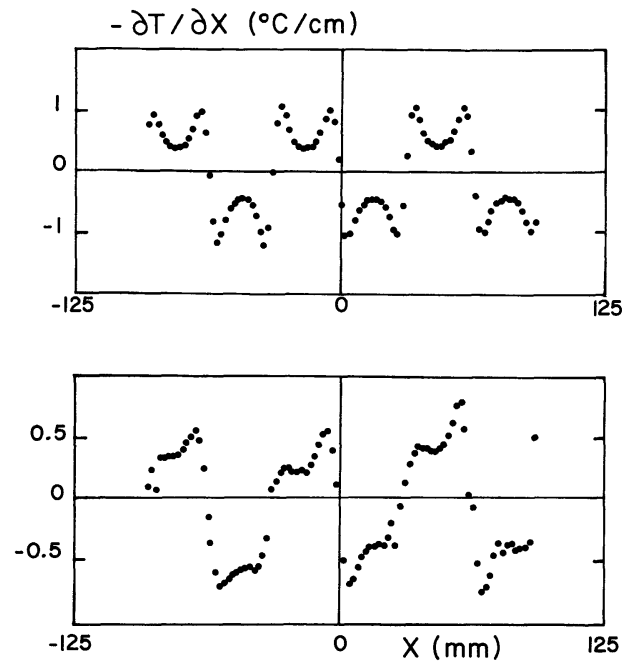

\begin{tabular}{|lllllll|}
\hline$\Uparrow$ & $\uparrow$ & $\uparrow$ & $\downarrow$ & $\uparrow$ & $\downarrow$ & $\uparrow$ \\
\hline$\uparrow$ & $\downarrow$ & $\uparrow$ & $\downarrow$ & $\uparrow$ & $\downarrow$ & $\uparrow$ \\
\hline
\end{tabular}

Fig. 4 - Strioscopy $X$-profiles for experiments N5. Top : horizontal gradient of temperature at mid-depth of the upper layer ; center : same for the lower layer; bottom: schematic interpretation in terms of uprising and downwelling currents.

$Y Z$-plane as well. This was done using aluminum flakes as velocity tracers. Figure 5 shows that four rolls are present in each layer (experiment Q3). The rolls are superposed. Color-coded pictures give, in addition, the sense of motion. They show that these transverse rolls are also «thermally coupled » with a downwelling in the center of the box in both layers,

Table II. - Experimental parameters.

\begin{tabular}{|c|c|c|c|c|c|}
\hline & & N3 & N5 & Q3 & P5 \\
\hline$\Delta T$ & temperature difference $\left({ }^{\circ} \mathrm{C}\right)$ & 20.8 & 7.2 & 8.3 & 8.4 \\
\hline$T_{\text {top }}$ & top plate temperature $\left({ }^{\circ} \mathrm{C}\right)$ & 15.2 & 21.8 & 30.2 & 22.1 \\
\hline$T_{\text {bot }}$ & bottom plate temperature $\left({ }^{\circ} \mathrm{C}\right)$ & 36.0 & 29.0 & 38.6 & 30.4 \\
\hline $\bar{\nu}_{\text {top }}$ & $\begin{array}{l}\text { viscosity of top layer }\left(10^{-6} \mathrm{~m}^{2} / \mathrm{s}\right) \\
\text { viscosity of bottom layer }\left(10^{-6} \mathrm{~m}^{2} / \mathrm{s}\right)\end{array}$ & $\begin{array}{l}530 \\
370\end{array}$ & $\begin{array}{l}510 \\
600\end{array}$ & $\begin{array}{l}425 \\
200\end{array}$ & $\begin{array}{l}500 \\
530\end{array}$ \\
\hline$R a_{\text {top }}$ & Rayleigh number top layer & 34000 & 12000 & 17000 & 14500 \\
\hline$R a_{\text {bot }}$ & Rayleigh number bottom layer & 16000 & 3400 & 12000 & 4500 \\
\hline $\begin{array}{l}M a_{\text {top }} \\
M a_{\text {bot }}\end{array}$ & $\begin{array}{l}\text { Marangoni number top layer } \\
\text { Marangoni number bottom layer }\end{array}$ & $\begin{array}{l}-700 \\
-540\end{array}$ & $\begin{array}{l}-280 \\
-110\end{array}$ & $\begin{array}{l}-380 \\
-400\end{array}$ & $\begin{array}{l}-330 \\
-150\end{array}$ \\
\hline $\begin{array}{l}\alpha \Delta T \\
G \\
C r\end{array}$ & $\begin{array}{l}\Delta \rho \\
\text { Bond number } \\
\text { Crispation number }\end{array}$ & & $\begin{array}{l}\sim 1 \\
\sim 7 \\
\sim 1\end{array}$ & & \\
\hline
\end{tabular}

$R a_{i}=\frac{\alpha_{i} \Delta T_{i} d_{i}^{3} g}{\kappa_{i} \bar{\nu}_{i}} ; M a_{i}=\frac{\Delta T_{i} d_{i} \sigma}{\rho_{i} \bar{\nu}_{i} \kappa_{i}}$ (where $i=$ top or bot) $; G \simeq \frac{g \Delta \rho d^{2}}{4 S} ; C r \simeq \frac{2 \rho \nu \kappa}{S d} ;$ Both layers are $25 \mathrm{~mm}$ thick in all the experiments. 


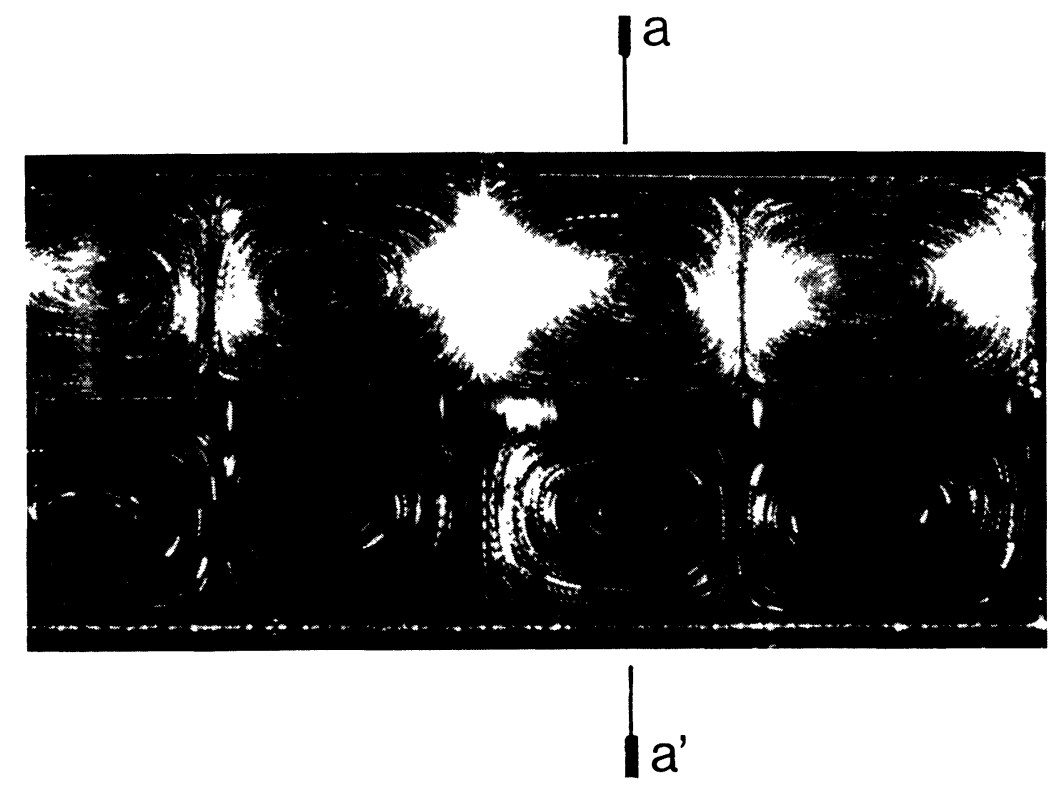

Fig. 5 - A velocity map in a $Y Z$ plane for experiment Q3. In each layer, four rolls are seen to occupy the width of the tank. The rolls are superposed. The illuminated slice of fluid falls in the middle of a roll with axis parallel to the short side of the tank. The light source is hatched with alternating black and white periods. Each period is $4 \mathrm{~s}$ long. There are 5 white periods. Line $\mathrm{aa}^{\prime}$ is the position of the vertical profile shown in figure 6.

for this experiment. We have measured velocities from the color-coded streak photographs. Figure 6

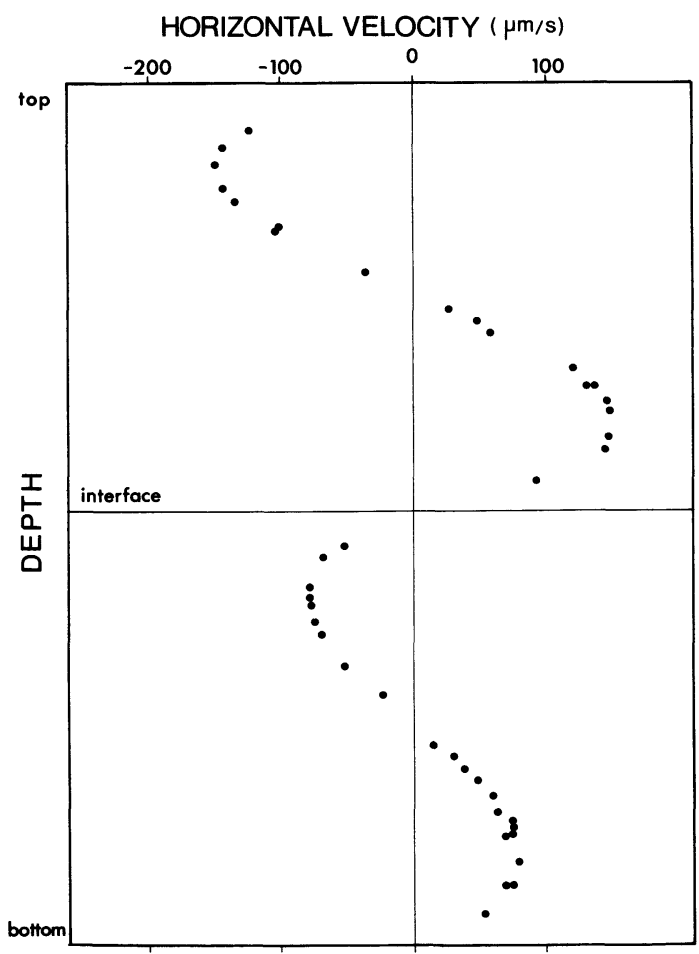

Fig. 6 - Vertical profile of the horizontal velocity derived from streak photographs such as the one shown in figure 5 (experiment Q3). The profile is taken in the middle of a pair of superposed rolls (line $\mathrm{aa}^{\prime}$ of Fig. 5). Note that the velocity is nearly zero at the interface, and that the rolls turn in the same counterclockwise sense. gives a vertical profile of the horizontal velocity in the middle of a pair of superposed rolls. The interface is seen by the fluids almost as the rigid boundaries of the top and bottom. This is quite in contrast with the « mechanical coupling » case where the interface is a region of minimum shear, and maximum horizontal velocity.

At this point, we have shown that «thermal coupling » is the preferred mode of coupling for the arrangement of fluids that we use in the lab. Since this is in contradiction with the available numerical results, we investigate in the next section what might be the reason for the disagreement.

\section{Discussion.}

There are, of course, several differences between the experimental conditions in the lab and the cases treated numerically. None of them is a priori capable of explaining the difference in behaviour that we observe. However, because of this difference, further checks are required. This section presents and discusses some of the checks we have performed.

INITIAL CONDITIONS. - We usually start convection by warming up the bottom plate. A thermal boundary layer develops, becomes unstable, and yields «plumes » that rise and strike the interface. Convection then starts in the upper layer. This scenario might be thought to favor «thermal coupling ». To check whether these initial conditions are responsible for the disagreement between the numerical and laboratory experiments, we artificially forced convection to start in the « mechanical coupling » mode. 
Chen and Whitehead [12] were the first to propose a set-up to induce a given convective pattern : they shine a strong light on a grid that sits on top of their transparent convection tank, while slowly building up the temperature drop across the fluid layer. This causes hot rising currents to form where the grid lets light through.

We slightly modified Chen and Whitehead's technique by placing the grid vertically next to the long wall of the tank, and shining the heating light from the side. Two types of grids were used : one for which slots for the upper layer are in opposition of phase with slots for the lower layer, to induce "mechanical coupling"; and one where slots for the two layers lie on the same vertical, to induce «thermal coupling ». We checked that our over-all procedure was efficient by successfully inducing 12 thermally coupled rolls in the length of the tank when only 8 to 10 would naturally have formed. Figure 7 shows the time evolution of the system when "mechanical coupling " is initiated. The first profiles indicate that rolls are indeed « mechanically coupled» at the beginning. But the next profiles show that they change to "thermal coupling" in about 1000 seconds (the "overturn time»- or typical time of rotation for a roll - is of the order of
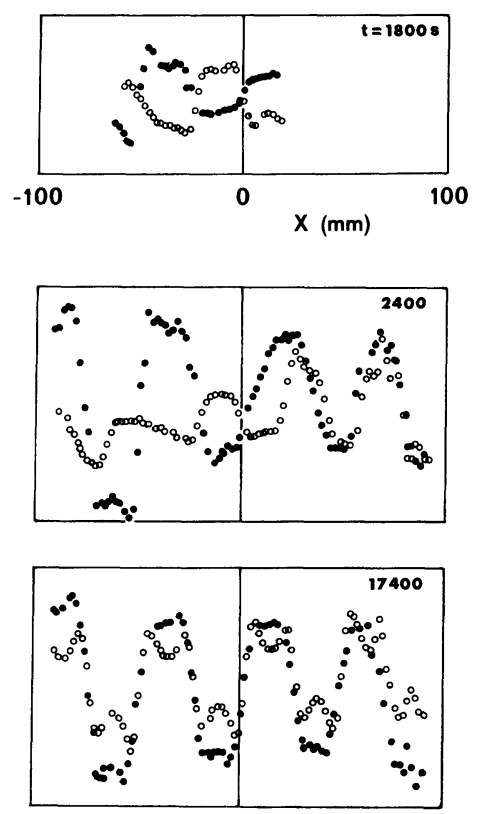

Fig. 7 - Time-evolution from artificially induced « mechanical » coupling to « thermal » coupling (experiment P5), as seen by strioscopy. Time, as counted from the start of the building of the temperature drop across the layers, is given in seconds. Two $X$-profiles of the horizontal gradient of temperature are given at each time ; both are taken at mid-depth : one in the upper layer (open circles), and the other one in the lower layer (filled circles). Note the transformation from " out of phase " profiles at the top to « in phase » profiles.
$500 \mathrm{~s}$ for this run). We conclude that «mechanical coupling $»$ is in fact unstable in the lab.

DEFORMATION OF THE INTERFACE. - The deformation of the interface is not explicitly taken into account in the numerical experiments of Cserepes and Rabinowicz [4]. This is why we used two fluids with a large density contrast, so that the amplitude of the deformation would be negligible in the lab as well ( $\alpha \Delta T / \Delta \rho$ small; see Tab. II). In order to check the validity of this assumption, we a posteriori measured the deformation of the interface. A laser beam is directed onto the interface, and the deflection of the reflected spot is monitored while the tank is moved in the $Y$ direction. The profile thus obtained is shown in figure 8 for experiment $Q 3$. It can be interpreted as the reflection from an almost sinusoïdal interface. The amplitude of the deformation is $0.12 \mathrm{~mm}$, which is to be compared with the $25 \mathrm{~mm}$-thickness of each layer.

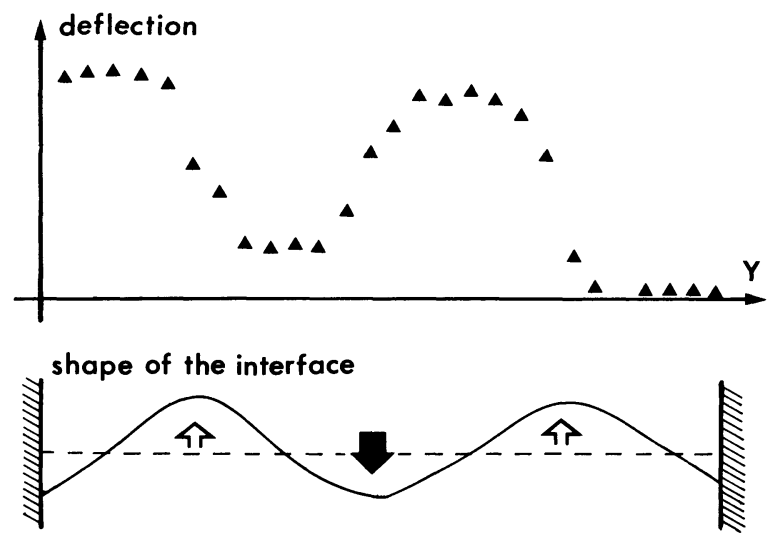

Fig. 8 - Top : deflection of a spot reflected off the interface as a function of $Y$, for experiment Q3. Bottom : deduced shape of the interface. The amplitude of the deformation is $0.12 \mathrm{~mm}$. The arrows show the sense of motion in the fluids as obtained from figure 5.

A rough estimate of the dynamical importance of the tilt of the interface is obtained by comparing the horizontal temperature gradient it produces to the horizontal gradient associated with the rolls at the interface. The latter, on the average, is the temperature difference between a hot uprising and a cold downwelling, divided by the width of a roll : it scales as $\Delta T / d$. The tilt of the interface lifts the interface temperature up to the same horizontal level as a region that is $2 \varepsilon$ above the interface (where $\varepsilon$ is the amplitude of the deformation). The vertical temperature gradient in the boundary layer is $\Delta T / 4 \delta$, where $\delta$ is the thickness of the boundary layer. The temperature difference between two points on the same horizontal is thus $2 \varepsilon(\Delta T / 4 \delta)$, and the temperature gradient $(\varepsilon / \delta) \times(\Delta T / d)$. For 
the low Rayleigh numbers considered here, the boundary layer thickness $\delta$ is at least $6 \mathrm{~mm}$. Therefore the relative importance of the interface deformation is probably no more than $\varepsilon / \delta=$ $0.12 \mathrm{~mm} / 6 \mathrm{~mm}=2 \%$. We conclude that the neglect of the deformation of the interface in the numerical experiments is not responsible for the reported discrepancy. Furthermore, the deformation of the interface was included in the marginal stability analyses of Richter and Johnson [3] and Honda [7] ; yet they both find « mechanical coupling » to be the preferred mode (with free slip boundaries).

INTERFACIAL TENSION. - Glycerol and silicon oil are immiscible. Surface tension is therefore present at the interface. It is well known that surface tension which varies with temperature can induce convective motions (the so-called Bénard or Marangoni instability). These effects might influence the style of coupling. We now show that interfacial tension effects appear to be negligible in our experiments.

The first indication of the unimportance of surface tension could come from the sense of deformation of the interface. We have reported in figure 8 the sense of motion deduced from the streak photographs. It can be seen that the interface is deflected upwards over uprising currents. This is the sense of deflection expected for buoyancy-dominated Rayleigh-Bénard convection [13]. If surface tension were the dominant mechanism, the deflection would have the opposite sign $[14,15]$. At least, this would be the case if interfacial tension were a decreasing function of temperature, as is the case for most fluids. However, we measured the tension at the interface between glycerol and silicon oil and found it to increase with temperature. The argument on the sense of the deflection is therefore not a safficient test.

A more quantitative assessment can be made by estimating the «capillary length » for the interface [16] :

$$
\lambda_{\mathrm{C}}=\sqrt{\frac{S}{\Delta \rho g}}=3 \mathrm{~mm}
$$

where $S$ is the interfacial tension, and $\Delta \rho$ the density difference. The competition between surface tension and buoyancy for controlling the shape of the interface is best described by a «thermal capillary length » [17] defined as :

$$
\lambda_{\mathrm{T}}=\lambda_{\mathrm{C}} \sqrt{\frac{|\sigma| / S}{\alpha}}=9 \mathrm{~mm}
$$

where $-\sigma$ is the temperature derivative of the interfacial tension, and $\alpha$ the average coefficient of thermal expansion (see Tab. I).

Both lengths are quite smaller than the depth of each layer, which is also the characteristic dimension of the rolls. This is a good indication that interfacial tension effects are small. The force balance at the interface can be writen as [16] :

$$
\begin{aligned}
\lambda\left[p^{(1)}-p^{(2)}-S\left(\frac{1}{R}+\frac{1}{R^{\prime}}\right)\right] n_{i}= & \\
& =\left(\tau_{i k}^{\prime(1)}-\tau_{i k}^{\prime(2)}\right) n_{k}+\frac{\partial S}{\partial X_{i}}
\end{aligned}
$$

where the superscript refers to one of the two liquids, $\tau^{\prime}$ is the deviatoric stress tensor, $p$ is the pressure, and $R$ and $R^{\prime}$ are the principal radii of curvature of the interface, whose normal is $n$. The balance in the horizontal direction $X$ reduces to :

$$
\mu^{(1)} \frac{\partial u^{(1)}}{\partial Z}-\mu^{(2)} \frac{\partial u^{(2)}}{\partial Z}=\frac{\mathrm{d} S}{\mathrm{~d} T} \frac{\partial T}{\partial X}
$$

where $\mu$ is the dynamic viscosity and $u$ the horizontal velocity. Using the values of material properties from table I, estimates for the temperature gradient from figure 4 , and estimates for the velocity gradient from figure 6 , we find that :

$$
\mu \frac{\partial u}{\partial Z}=2 \times 10^{-2} \mathrm{~N} / \mathrm{m}^{2}
$$

and

$$
\frac{\mathrm{d} S}{\mathrm{~d} T} \frac{\partial T}{\partial X}=10^{-2} \mathrm{~N} / \mathrm{m}^{2}
$$

The two terms are of the same order, and horizontal interfacial tension might therefore play a role. However, when the $(\mathrm{d} S / \mathrm{d} T)(\partial T / \partial X)$ term is included in the numerical experiments, no change in the coupling between the two layers is observed until that term is about one hundred times larger than the viscous terms [18].

All arguments therefore tend to indicate that although interfacial tension is not really «negligible » in our experiments, it is probably not responsible in a simple way for the discrepancy that we observe between the laboratory and the numerical experiments.

A TENTATIVE ExPlanAtion. - In order for « thermal coupling » to take over « mechanical coupling », we need to invoke some kind of stress uncoupling at the interface. For example, if the interface was behaving as a very thin perfectly lubricating surface the shear stresses would not be transmitted from one layer to the other. This hypothesis is excluded from the velocity profile of figure 6 , which shows that the fluids see the interface more as a rigid boundary than a free-slip boundary. Then, could the interface be so viscous that it would appear as a rigid film for the two fluids? In favor of this possibility is the following observation : if we let the silicon oil layer be twice as thick as the glycerol layer, it is possible to induce convection in the thick layer while the thin one is not convecting; the active layer should then drag and 
entrain the passive layer in a « mechanical coupling » mode. On the contrary, we observe no entrainment of the subcritical layer. The interface behaves as a barrier for stress transmission. What then makes the interface be so «viscous »? We have noticed in the case of miscible fluids an entrainment phenomenon [9] : material from the lower layer is dragged by the upper one, and entrained at uprisings in the form of filaments. This indicates that the interface is submitted to large compressional stresses. If the interface is characterized by a large longitudinal viscosity, it could refuse to transmit the shear stresses from one layer to the other. Filaments cannot form with immiscible fluids, because surface tension prevents the formation of spike-like distorsions of the interface. We think that this indirect surface tension effect (which acts on a length scale much smaller than the characteristic roll dimensions) could inhibit longitudinal deformation of the interface. The very large equivalent interface viscosity that results would not be seen if the interface is only submitted to simple shear.

\section{Conclusion.}

We have investigated the structure of convection in a 2-layer system. In the lab we observe «thermal coupling » (where rolls are superposed, with uprising above uprising) to be the only stable mode. This is in contradiction with $2-\mathrm{D}$ and $3-\mathrm{D}$ numerical results obtained on the same problem $[4,7,8]$, where «mechanical coupling» (superposed rolls turn in opposite senses) was found to be preferred. We have conducted a number of tests to explain the discrepancy. These show that initial conditions, and deformation of the interface are not responsible for the reported disagreement. Interfacial tension also seems to be negligible. We propose a tentative mechanism, in which a large interfacial equivalent viscosity results from the inhibition of spike-like entrainment at the interface, due to interfacial tension acting on a very small length scale. This explanation might need revision as further tests become available. What will remain is the demonstration that "thermal coupling " is the only stable convective situation for the experimental conditions we chose, a somewhat unexpected finding.

\section{Acknowledgments.}

We wish to thank Gilbert Malfait who built our apparatus. We are thankful to Michel Rabinowicz and Lazlo Cserepes for their ongoing and friendly interaction with us. Luce Fleitout suggested the use of Chen and Whitehead's method. We are grateful to Jean-Claude Loulergue, Vincent Croquette, Christian Bayon, and Robert Reich for valuable help at diverse stages of this study. Neil Ribe and two anonymous reviewers brought very significant improvments to this paper. This work was supported by CNRS ATP « Dynamique des Fluides Géophysiques et Astrophysiques ». S. M. acknowledges support from Ministero della Publica Istruzione (Italia). Contribution CNRS-INSU-DBT $\mathrm{n}^{\circ} 9$, Thème «Couplages ».

\section{References}

[1] Davies, G. F., J. Geophys. Res. 89 (1984) 60176040.

[2] Richter, F. M. and McKenzie, D. P., J. Geophys. Res. 86 (1981) 6133-6142;

Olson, P., J. Geophys. Res. 13 (1984) 11293-11302.

[3] Richter, F. M. and Johnson, C. E., J. Geophys. Res. 79 (1974) 1635-1639.

[4] Cserepes, L. and Rabinowicz, M., Earth Planet. Sci. Lett. 76 (1985) 193-207.

[5] Renardy, Y. and Joseph, D. D., Phys. Fluids 28 (1985) 788-793.

[6] Loulergue, J. C., Manneville, P. and Pomeau, Y., J. Phys. D 14 (1981).

[7] HondA, S., Bull. Earthquake Res. Inst. 57 (1982) 273-302.

[8] Cserepes, L., Rabinowicz, M. and RosembergBorot, C., J. Geophys. Res., in press (1988).

[9] NataF, H. C. and Moreno, S. (abstract), 23rd meeting of I.A.S.P.E.I. (Tokyo) 1985.

[10] Nataf, H. C., Froidevaux, C., Levrat, J. L. and RABInOwiCZ, M., J. Geophys. Res. 86 (1981) 6143-6154.
[11] RabinowiCZ, Michel, personal commun. (1987).

[12] Chen, M. M. and Whitehead, J. A., J. Fluid Mech. 31 (1968) 1-15.

[13] Jeffreys, H. H., Q. J. Mech. Appl. Math. 4 (1951) 283.

[14] Cerisier, P., Jamond, C., Pantaloni, J. et CharMET, J. C., J. Phys. France 45 (1984) 405-411.

[15] Perez-Garcia, C., Pantaloni, J., Occelli, R. and Cerisier, P., J. Phys. France 46 (1985) 2047-2051.

[16] Landau, L. et LifChitz, Mécanique des fluides (MIR, Moscou) 1971.

[17] Loulergue, J. C., Etude des déformations des interfaces induites par effet Marangoni dans un système diphasique. Application à la conversion d'images infrarouges en visibles, Thèse d'Etat, Univ. Paris-Sud (1983).

[18] RABINOWiCZ, Michel and CSEREPES, Lazlo, personal commun. (1987). 